\title{
AVERAGING THEORY FOR DISCONTINUOUS PIECEWISE DIFFERENTIAL SYSTEMS
}

\author{
JAUME LLIBRE ${ }^{1}$, ANA C. MEREU ${ }^{2}$ AND DOUGLAS D. NOVAES ${ }^{3}$
}

\begin{abstract}
We develop the averaging theory of first and second order for studying the periodic solutions of discontinuous piecewise differential systems in arbitrary dimension and with an arbitrary number of systems with the minimal conditions of differentiability. We do two applications.
\end{abstract}

\section{INTRODUCTION AND STATEMENT OF THE MAIN RESULTS}

1.1. Introduction. The discontinuous differential systems, i.e. differential equations with discontinuous right-hand sides, is a subject that has been developed very fast these last years. It has become certainly one of the common frontiers between Mathematics, Physics and Engineering. Thus certain phenomena in control systems [2], impact and friction mechanics [7], nonlinear oscillations $[1,18]$, economics $[12,13]$, and biology $[3,15]$, are the main sources of motivation of their study, see for more details Teixeira [20]. A recent review appears in [22].

The knowledge of the existence or not of periodic solutions is very important for understanding the dynamics of differential systems. One of good tools for study the periodic solutions is the averaging theory, see for instance the books of Sanders and Verhulst [19] and Verhulst [21]. We point out that the method of averaging is a classical and matured tool that provides a useful means to study the behaviour of nonlinear smooth dynamical systems. The method of averaging has a long history that starts with the classical works of Lagrange and Laplace who provided an intuitive justification of the process. The first formalization of this procedure was given by Fatou in 1928 [10]. Very important practical and theoretical contributions in the averaging theory were made by Krylov and Bogoliubov [6] in the 1930s and Bogoliubov [5] in 1945. The principle of averaging has been extended in many directions for both finite- and infinite-dimensional differentiable systems. The classical results for studying the periodic orbits of differential systems need at least that those systems be of class $\mathcal{C}^{2}$. Nevertheless Buica and Llibre [9] extended the averaging theory for studying periodic orbits to continuous differential systems using mainly the Brouwer degree theory. Recently in [16] developed the averaging theory for studying periodic orbits to discontinuous differential systems.

1.2. Preliminaries. In the following we define the necessary elements for the statement of our main results.

Let $D$ be an open subset of $\mathbb{R}^{d}$ and $I=[0, T)$ an interval of $\mathbb{R}$. We consider a finite set of ODEs

$$
x^{\prime}(t)=f^{n}(t, x), \quad(t, x) \in I \times D \quad \text { for } \quad n=1,2, \ldots, M,
$$

2010 Mathematics Subject Classification. 37G15, 37C80, 37C30

Key words and phrases. periodic solution, averaging theory, discontinuous differential system, non-smooth differential systems. 
where $f^{n}: I \times D \rightarrow \mathbb{R}^{d}$ is a continuous function. Here the prime denotes derivative with respect to the time $t$. Let $\left(S_{n}\right)$ be a set of open disjoints subset of $I \times D$ for $n=1,2, \ldots, M$. We suppose that the boundaries of each $S_{n}$ are $\mathcal{C}^{k}$ embedded piecewise hypersurfaces with $k \geq 1$. Furthermore the union of all boundaries, denoted by $\Sigma$, and all $S_{n}$ together cover $I \times D$. So we define a $M$-Discontinuous Piecewise Differential System, or simply a $M-D P D S$ as

$$
x^{\prime}(t)=f(t, x)=\left\{\begin{array}{cc}
f^{1}(t, x), & (t, x) \in \overline{S_{1}}, \\
f^{2}(t, x), & (t, x) \in \overline{S_{2}}, \\
& \vdots \\
f^{M}(t, x), & (t, x) \in \overline{S_{M}},
\end{array}\right.
$$

where $\overline{S_{k}}$ denotes the closure of $S_{k}$ in $D$.

Remark 1. Later on in this work we shall assume that the functions $f^{n}$ are Lipschitz, and the boundaries are piecewise $\mathcal{C}^{k}$ embedded hypersurfaces with either $k \geq 1$ or $k \geq 2$. However the theory described in the following is developed without these assumptions.

Let $A$ be a subset of $I \times D$ and let $\chi_{A}(t, x)$ be the characteristic function defined as

$$
\chi_{A}(t, x)= \begin{cases}1 & \text { if } \quad(t, x) \in A \\ 0 & \text { if } \quad(t, x) \notin A .\end{cases}
$$

So system (2) can be written as

$$
x^{\prime}(t)=f(t, x)=\sum_{n=1}^{M} \chi_{\overline{S_{n}}}(t, x) f^{n}(t, x), \quad(t, x) \in I \times D .
$$

Remark 2. The Filippov convention for the flow of system (3) passing through a point $p \in \Sigma$ does not depend on the value $f(p)$. Therefore we can define $f(p)$ for $p \in \Sigma$ in any way, also multivalued as usual. Details on Filippov conventions for differential equations with discontinuous righthand side can be found in the book of Filippov [11].

A point $p \in \Sigma$ is called a generic point of discontinuity if there exists a neighborhood $G_{p}$ of $p$ such that $\mathcal{S}_{p}=G_{p} \cap \Sigma$ is a $\mathcal{C}^{k}$ embedded hypersurface. In this case we can always assume that the hypersurface $\mathcal{S}_{p}$ splits $G_{p} \backslash \mathcal{S}_{p}$ in two disconnected regions, namely $G_{p}^{+}$and $G_{p}^{-}$.

Let $p$ be a generic point of discontinuity. We denote $f_{p}^{+}=\left.f\right|_{G_{p}^{+}}$and $f_{p}^{-}=\left.f\right|_{G_{p}^{-}}$, which are continuous vector fields. So we define $l(p)$ as the segment connecting the vectors $f_{p}^{+}(p)$ and $f_{p}^{-}(p)$, see Figures 1 and 2. The sets $G_{p}, G_{p}^{+}$and $G_{p}^{-}$are not uniquely defined. Nevertheless, it is easy to see that $f_{p}^{+}(p), f_{p}^{-}(p)$, and $l(p)$ do not depend of this choice.

An embedded hypersurface $\mathcal{S} \subset \Sigma$ can be decomposed as the union of the closure of its crossing region $\Sigma^{c}(\mathcal{S})$ (see Figure 1), and its sliding region $\Sigma^{s}(\mathcal{S}$ ) (see Figure 2), which are defined as

$$
\Sigma^{c}(\mathcal{S})=\left\{p \in \mathcal{S}: l(p) \cap T_{p} \mathcal{S}=\emptyset\right\} \quad \text { and } \quad \Sigma^{s}(\mathcal{S})=\left\{p \in \mathcal{S}: l(p) \cap T_{p} \mathcal{S} \neq \emptyset\right\}
$$

where as usual $T_{p} \mathcal{S}$ denotes the tangent space of $\mathcal{S}$ at the point $p$.

If $\mathcal{S}=h^{-1}(0)$ for some $\mathcal{C}^{1}$ function $h: I \times D \rightarrow \mathbb{R}$ which has 0 as a regular value, then the above definitions can be written as

$$
\begin{aligned}
& \Sigma^{c}(\mathcal{S})=\left\{p \in \mathcal{S}:\left\langle\nabla h(p), f^{+}(p)\right\rangle\left\langle\nabla h(p), f^{-}(p)\right\rangle>0\right\} \quad \text { and } \\
& \Sigma^{\mathcal{S}}(\mathcal{S})=\left\{p \in \mathcal{S}:\left\langle\nabla h(p), f^{+}(p)\right\rangle\left\langle\nabla h(p), f^{-}(p)\right\rangle<0\right\} .
\end{aligned}
$$



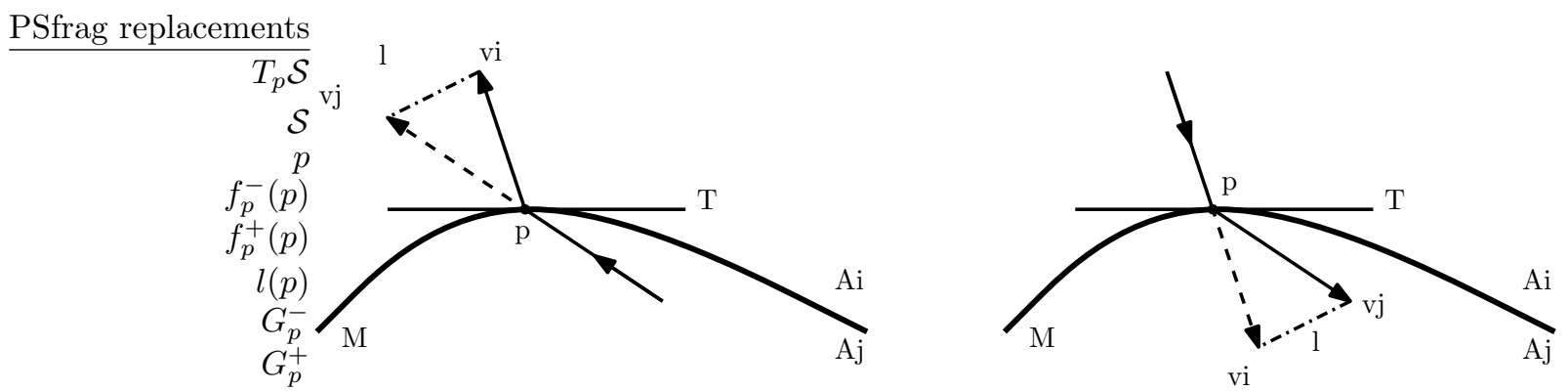

FIGURE 1. Crossing region of $\mathcal{S}: \Sigma^{c} \mathcal{S}$.

PSfrag replacements

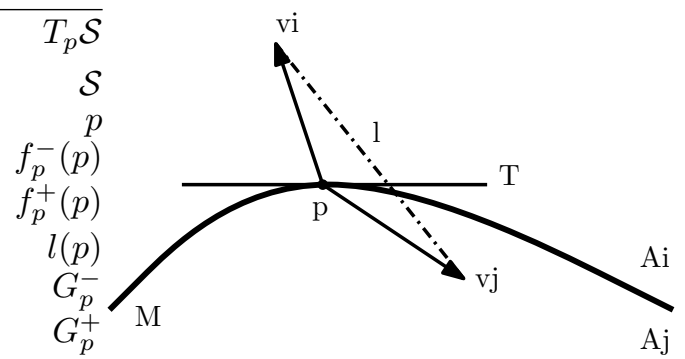

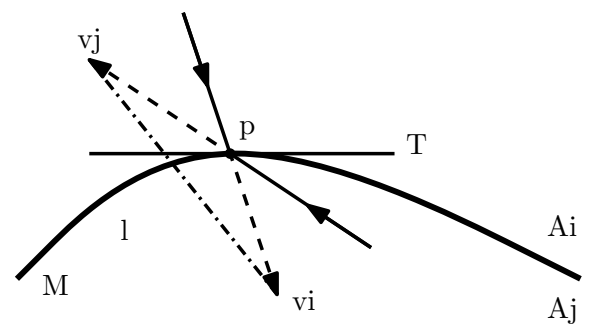

FiguRE 2. Sliding region of $\mathcal{S}: \Sigma^{s} \mathcal{S}$.

The crossing region $\Sigma^{c}$ is defined as the generic points of discontinuity $p$ such that $p \in \Sigma^{c}\left(\mathcal{S}_{p}\right)$. Analogously, we define the sliding region $\Sigma^{s}$.

Let $\varphi_{f^{n}}(t, q)$ be the solution of system (1) passing through the point $q \in \S_{n}$ at time $t=0$, i.e. $\varphi_{f^{n}}(o, q)=q$. The local solution $\varphi_{f}(t, q)$ of system (3) passing through a point $p \in \Sigma^{c}$ at time $t=0$ is given by the Filippov convention, i.e. for $p \in \Sigma^{c}$ such that $l(p) \subset G_{p}^{+}$and taking the origin of time at $p$, the trajectory through $p$ is defined as $\varphi_{f}(t, p)=\varphi_{f_{p}^{-}}(t, p)$ for $t \in I_{p} \cap\{t<0\}$, and $\varphi_{f}(t, p)=\varphi_{f_{p}^{+}}(t, p)$ for $t \in I_{p} \cap\{t>0\}$. For the case $l(p) \subset G_{p}^{-}$the definition is the same reversing the time. Here $I_{p}$ is an open interval having the 0 in its interior.

The following proposition gives a condition for the existence and uniqueness of solutions of system (3).

Proposition 1. For every point $p \in \Sigma^{c}$ there is a solution passing either from $G_{p}^{-}$into $G_{p}^{+}$, or from $G_{p}^{+}$into $G_{p}^{-}$, and uniqueness in not violated.

For a proof of Proposition 1 see Corollary 1 of section 10 of chapter 2 of [11].

Remark 3. The Lipschitz condition together with Proposition 1 imply, for system (3), the global uniqueness of solutions whose reach the set of discontinuity only at points of $\Sigma^{c}$.

1.3. Statement of the main results. We consider the following DPDS.

$$
x^{\prime}(t)=\varepsilon F_{1}(t, x)+\varepsilon^{2} F_{2}(t, x)+\varepsilon^{3} R(t, x, \varepsilon),
$$


with

$$
\begin{aligned}
& F_{i}(t, x)=\sum_{j=1}^{M} \chi_{\overline{S_{j}}}(t, x) F_{i}^{j}(t, x), \quad \text { for } i=1,2, \text { and } \\
& R(t, x, \varepsilon)=\sum_{j=1}^{M} \chi_{\overline{S_{j}}}(t, x) R^{j}(t, x),
\end{aligned}
$$

where $F_{i}^{j}: \mathbb{R} \times D \rightarrow \mathbb{R}^{d}, R^{j}: \mathbb{R} \times D \times\left(-\varepsilon_{0}, \varepsilon_{0}\right) \rightarrow \mathbb{R}^{d}$ for $i=1,2$ and $j=1,2, \ldots, M$ are continuous functions, $T$-periodic in the variable $t$ and $D$ is an open subset of $\mathbb{R}^{d}$.

Remark 4. Here the family of sets $\mathcal{P}=\left\{S_{i}: i=1,2, \ldots, M\right\}$ provides a partition of $[0, T) \times D$. Clearly we can use the family $\mathcal{P}$ for producing a partition of the intervals $[k T,(k+1) T)$ for $k \in \mathbb{Z}$. Since the righthand side of system (4) is T-periodic, a partition of $\mathbb{R} \times D$ can be obtained naturally by considering $\mathbb{R}=\cup_{k \in \mathbb{Z}}[k T,(k+1) T)$.

We denote for $i=1,2$

$$
D_{x} F_{i}(t, x)=\sum_{j=1}^{M} \chi_{\overline{A_{j}}}(t, x) D_{x} F_{i}^{j}(t, x) .
$$

In order to state our main result we define the averaged functions $f_{1}, f_{2}: D \rightarrow \mathbb{R}^{d}$ as

$$
\begin{gathered}
f_{1}(z)=\int_{0}^{T} F_{1}(t, z) d t, \quad \text { and } \\
f_{2}(z)=\int_{0}^{T}\left(D_{z} F_{1}(t, z) y_{1}(t, z)+F_{2}(t, z)\right) d t
\end{gathered}
$$

where

$$
y_{1}(t, z)=\int_{0}^{t} F_{1}(s, z) d s .
$$

Our main results on the periodic orbits of DPDS (4) are given in the next two theorems. Their proofs use the Brouwer degree theory for finite dimensional spaces (see the appendix for a definition of the Brouwer degree $\left.d_{B}(f, V, 0)\right)$.

Theorem A (First order averaging theorem for DPDS). Assume the following conditions.

(Ha1) For $i=1,2$ and $j=1,2, \ldots, M$, the continuous functions $F_{i}^{j}$ and $R_{i}^{j}$ are locally Lipschitz with respect to $x$, and $T$-periodic with respect to the time $t$. Furthermore, for $j=1,2, \ldots, M$, the boundaries of $S_{j}$ are piecewise $\mathcal{C}^{k}$ embedded hypersurfaces with $k \geq 1$.

(Ha2) There exists an open bounded set $C \subset D$ such that, for $|\varepsilon|>0$ sufficiently small, every orbit starting in $C$ reaches the set of discontinuity only at its crossing regions (crossing hypothesis).

(Ha3) For $a \in C$ with $f_{1}(a)=0$, there exist a neighbourhood $U \subset C$ of a such that $f_{1}(z) \neq 0$ for all $z \in \bar{U} \backslash\{a\}$ and $d_{B}\left(f_{1}, U, 0\right) \neq 0$.

Then for $|\varepsilon|>0$ (or $\varepsilon>0$ ) sufficiently small, there exists a $T$-periodic solution $x(t, \varepsilon)$ of system (4) such that $x(0, \varepsilon) \rightarrow$ a as $\varepsilon \rightarrow 0$.

Theorem A is proved in section 3 .

Theorem B (Second order averaging theorem for DPDS). Suppose that $f_{1}(z) \equiv 0$. In addition to the hypotheses of Theorem A assume the following conditions. 
(Hb1) For $j=1,2, \ldots, M$, the functions $F_{1}^{j}(t, \cdot)$ are of class $\mathcal{C}^{1}$ for all $t \in \mathbb{R} ;$ for $i=1,2$ and $j=1,2, \ldots, M$, the functions $D_{x} F_{1}^{j}$ are locally Lipschitz with respect to $x$. Furthermore, for $j=1,2, \ldots, M$, the boundaries of $S_{j}$ are piecewise $\mathcal{C}^{k}$ embedded hypersurfaces with $k \geq 2$.

(Hb2) For $(t, z) \in \Sigma$, if $\left(s, y_{1}(t, z)\right) \in T_{(t, z)} \Sigma$ then $s=0$.

(Hb3) For $a \in C$ with $f_{2}(a)=0$, there exist a neighborhood $U \subset C$ of a such that $f_{2}(z) \neq 0$ for all $z \in \bar{U} \backslash\{a\}$ and $d_{B}\left(f_{2}, U, 0\right) \neq 0$.

Then for $|\varepsilon|>0$ sufficiently small, there exists a T-periodic solution $x(t, \varepsilon)$ of system (4) such that $x(0, \varepsilon) \rightarrow$ as $\varepsilon \rightarrow 0$.

Theorem B is also proved in section 3 .

1.4. Discontinuous perturbation of planar linear centers. In this subsection we show how to use the Theorems A and B for studying the linear centers perturbed by DPDS systems having the set of discontinuity composed by rays passing through the origin of coordinates. In other words we shall show that the hypothesis of crossing (Ha2) of both theorems and the hypothesis $(\mathrm{Hb2})$ of Theorem B always hold for such systems after a change of variables and a rescaling of the time.

Let $M$ be a positive integer greater than 1 , let $\alpha=\left(\alpha_{1}, \alpha_{2}, \cdots, \alpha_{M}\right) \in \mathbb{T}^{M}$ (M-Torus) be a $M$-tuple of angles such that $0 \leq \alpha_{1}<\alpha_{2}<\cdots<\alpha_{M}<2 \pi$ and let $\mathcal{X}=\left(X_{1}, X_{2}, \cdots, X_{M}\right)$ be a $M$-tuple of locally Lipschitz vector fields defined on an open neighborhood $D \subset \mathbb{R}^{2}$ of the origin.

We define the set of discontinuity $\Sigma=\bigcup_{i=1}^{M} L_{i}$, where $L_{i}$ for $i=1,2, \ldots, M$, is the intersection between the ray starting at the origin and passing through the point $\left(\cos \alpha_{i}, \sin \alpha_{i}\right)$ with the set $D$. We note that the set $\Sigma$ splits the set $D \backslash \Sigma \subset \mathbb{R}^{2}$ in $M$ disjoint open sectors. We denote the sector delimited by $L_{i}$ and $L_{i+1}$ by $C_{i}$ for $i=1,2, \ldots, M$.

Now let $Z_{\mathcal{X}, \alpha}(x, y)$ be the DPDS defined in $D$ as

$$
Z_{\mathcal{X}, \alpha}(x, y)=X_{i}(x, y) \quad \text { if } \quad(x, y) \in C_{i} .
$$

Let $\alpha$ be a $M$-tuple of angles, and let $\mathcal{X}$ and $\mathcal{Y}$ be two $M$-tuples of vector fields. In this section we shall study the following DPDS.

$$
(\dot{x}, \dot{y})=(y,-x)+\varepsilon Z_{\mathcal{X}, \alpha}(x, y)+\varepsilon^{2} \varepsilon Z_{\mathcal{Y}, \alpha}(x, y) .
$$

Now the dot denotes derivative with respect to the time $t$.

Using the polar coordinates $x=r \cos \theta$ and $y=r \sin \theta$, system (7) becomes

$$
(\dot{\theta}, \dot{r})=(-1,0)+\varepsilon A(\theta, r)+\varepsilon^{2} B(\theta, r),
$$

where $A$ and $B$ are DPDS with the set of discontinuity $\tilde{\Sigma}$ being the union of the rays $\left\{\left(\alpha_{i}, r\right)\right.$ : $r>0\}$ for $i=1,2, \ldots, M$. Moreover, for $\theta \in[0,2 \pi), A(\theta, r)=A_{i}(\theta, r)$ and $B(\theta, r)=B_{i}(\theta, r)$ if $\alpha_{i} \leq \theta \leq \alpha_{i+1}$ for $i=1,2, \ldots, M$, where $\alpha_{M+1}=\alpha_{1}$, and

$$
\begin{array}{r}
A_{i}(\theta, r)=\left(\frac{1}{r}\left(X_{i}^{2}(r \cos \theta, r \sin \theta) \cos \theta-X_{i}^{1}(r \cos \theta, r \sin \theta) \sin \theta\right),\right. \\
\left.X_{i}^{1}(r \cos \theta, r \sin \theta) \cos \theta+X_{i}^{2}(r \cos \theta, r \sin \theta) \sin \theta\right),
\end{array}
$$


and

$$
\begin{aligned}
B_{i}(\theta, r)= & \left(\frac{1}{r}\left(Y_{i}^{2}(r \cos \theta, r \sin \theta) \cos \theta-Y_{i}^{1}(r \cos \theta, r \sin \theta) \sin \theta\right),\right. \\
& \left.Y_{i}^{1}(r \cos \theta, r \sin \theta) \cos \theta+Y_{i}^{2}(r \cos \theta, r \sin \theta) \sin \theta\right) .
\end{aligned}
$$

Here $X_{i}=\left(X_{i}^{1}, X_{i}^{2}\right)$ and $Y_{i}=\left(Y_{i}^{1}, Y_{i}^{2}\right)$ for $i=1,2, \ldots, M$.

In the sector $\alpha_{i} \leq \theta \leq \alpha_{i+1}$ system (8), taking the time $\theta$ instead $t$ can be written as the differential equation

$$
\frac{d r}{d \theta}=\frac{\dot{r}}{\dot{\theta}}=\frac{\varepsilon A_{i}^{2}(\theta, r)+\varepsilon^{2} B_{i}^{2}(\theta, r)}{-1+\varepsilon A_{i}^{1}(\theta, r)+\varepsilon^{2} B_{i}^{1}(\theta, r)} .
$$

Here $A_{i}=\left(A_{i}^{1}, A_{i}^{2}\right)$ and $B_{i}=\left(B_{i}^{1}, B_{i}^{2}\right)$ for $i=1,2, \ldots, M$. So system (8) and consequently system (7) becomes

$$
r^{\prime}=R(\theta, r, \varepsilon)
$$

where, for $i=1,2, \ldots, M, R(\theta, r, \varepsilon)=R_{i}(\theta, r, \varepsilon)$ if $\alpha_{i} \leq \theta \leq \alpha_{i+1}$, and

$$
\begin{aligned}
R_{i}(\theta, r, \varepsilon)= & -\varepsilon\left(X_{i}^{1}(r \cos \theta, r \sin \theta) \cos \theta+X_{i}^{2}(r \cos \theta, r \sin \theta) \sin \theta\right) \\
& -\varepsilon^{2}\left(\frac{1}{r}\left(X_{i}^{2}(r \cos \theta, r \sin \theta) \cos \theta-X_{i}^{1}(r \cos \theta, r \sin \theta) \sin \theta\right)\right. \\
& \cdot\left(X_{i}^{1}(r \cos \theta, r \sin \theta) \cos \theta+X_{i}^{2}(r \cos \theta, r \sin \theta) \sin \theta\right) \\
& \left.+\left(Y_{i}^{1}(r \cos \theta, r \sin \theta) \cos \theta+Y_{i}^{2}(r \cos \theta, r \sin \theta) \sin \theta\right)\right)+\mathcal{O}\left(\varepsilon^{3}\right) .
\end{aligned}
$$

Now the prime denotes derivative with respect to the time $\theta$.

Proposition 2. For system (9) the following statements hold.

(a) There exists an open bounded interval $C \subset(0, \infty)$ such that, for $|\varepsilon|>0$ sufficiently small, every orbit starting in $C$ reaches the set of discontinuity $\tilde{\Sigma}$ only at its crossing regions.

(b) If $\left(s, y_{1}\left(\alpha_{i}, r\right)\right) \in T_{\left(\alpha_{i}, r\right)} \tilde{\Sigma}$ then $s=0$ for $i=1,2, \ldots, M$.

Proof. Let $\tilde{\Sigma}_{i}=\left\{\left(\alpha_{i}, r\right): r>0\right\} \subset \tilde{\Sigma}$, and let $h_{i}(\theta, r)=\theta-\alpha_{i}$. Clearly $\tilde{\Sigma}=\cup_{i=1}^{M} \tilde{\Sigma}_{i}$ and $\tilde{\Sigma}_{i}=h_{i}^{-1}(0)$. In order to study the type of $\tilde{\Sigma}_{i}$, according to the definitions of Section 1.2, we compute

$$
\begin{aligned}
& \left\langle\nabla h_{i}\left(\alpha_{i}, r\right),\left(1, R_{i-1}\left(\alpha_{i}, r, \varepsilon\right)\right)\right\rangle\left\langle\nabla h_{i}\left(\alpha_{i}, r\right),\left(1, R_{i}\left(\alpha_{i}, r, \varepsilon\right)\right)\right\rangle= \\
& \left\langle(1,0),\left(1, R_{i-1}\left(\alpha_{i}, r, \varepsilon\right)\right)\right\rangle\left\langle(1,0),\left(1, R_{i}\left(\alpha_{i}, r, \varepsilon\right)\right)\right\rangle=1>0 .
\end{aligned}
$$

So $\tilde{\Sigma}$ has only crossing regions. Hence statement $(a)$ is proved.

Now assume that $\left(s, y_{1}\left(\alpha_{i}, r\right)\right) \in T_{\left(\alpha_{i}, r\right)} \tilde{\Sigma}$. It implies that

$$
0=\left\langle\nabla h_{i}\left(\alpha_{i}, r\right),\left(s, y_{1}\left(\alpha_{i}, r\right)\right)\right\rangle=\left\langle(1,0),\left(s, y_{1}\left(\alpha_{i}, r\right)\right)\right\rangle=s .
$$

So $s=0$, and statement $(b)$ is proved. 
1.5. Example 1. In the following example we solve a problem of type (7).

Consider $\alpha=(0, \pi / 2, \pi, 3 \pi / 2,2 \pi) \in \mathbb{T}^{4}$. Thus $L_{1}=\{(x, 0): x>0\}, L_{2}=\{(0, y): u>0\}$, $L_{3}=\{(x, 0): x<0\}$, and $L_{4}=\{(0, y): y<0\}$. Then for $i=1, \ldots, 4$ we have that $C_{i}$ is the first, second, third and fourth quadrants, respectively.

In this example we study the maximum number of limit cycles given by the averaging theory of first and second order, which can bifurcate from the periodic orbits of the linear center $\dot{x}=y$, $\dot{y}=-x$, perturbed inside the following class of linear DPDS:

$$
\dot{X}=Y_{i}(x, y) \quad \text { if } \quad(x, y) \in C_{i}, i=1, \ldots, 4
$$

where

$$
Y_{i}(x, y)=\left(\begin{array}{c}
y+\varepsilon P_{i}^{1}(x, y)+\varepsilon^{2} P_{i}^{2}(x, y) \\
-x+\varepsilon Q_{i}^{1}(x, y)+\varepsilon^{2} Q_{i}^{2}(x, y)
\end{array}\right),
$$

with $P_{i}^{1}(x, y)=a_{0 i}+a_{1 i} x+a_{2 i} y, P_{i}^{2}(x, y)=c_{0 i}+c_{1 i} x+c_{2 i} y, Q_{i}^{1}(x, y)=b_{0 i}+b_{1 i} x+b_{2 i} y$, $Q_{i}^{2}(x, y)=d_{0 i}+d_{1 i} x+d_{2 i}$ and $|\varepsilon|>0$ is a small parameter.

Let $\mathcal{A}$ denote the two conditions

$$
\begin{aligned}
& 4 a_{01}-4\left(a_{02}+a_{03}-a_{04}-b_{01}-b_{02}+b_{03}+b_{04}\right)=0 \quad \text { and } \\
& 2 a_{21}-2\left(a_{22}-a_{23}+a_{24}-b_{11}+b_{12}-b_{13}+b_{14}\right)+ \\
& \left(a_{11}+a_{12}+a_{13}+a_{14}+b_{21}+b_{22}+b_{23}+b_{24}\right) \pi=0 .
\end{aligned}
$$

Our results on the limit cycles of system (10) are stated in the next two propositions.

Proposition 3. For $|\varepsilon|>0$ sufficiently small and using Theorem A system (10) has at most 1 limit cycle for any chosen of parameters for $\mathcal{A}$ does not hold. Moreover we can find parameters $a_{i j}, b_{i j}, c_{i j}$, and $d_{i j}$ for $i=0,1,2$ and $j=1, \ldots, 4$ such that system (10) has exactly 0 or 1 limit cycle.

Proposition 4. For $|\varepsilon|>0$ sufficiently small and using Theorem B system (10) has at most 4 limit cycles for any chosen of parameters for which the two conditions of $\mathcal{A}$ holds. Moreover we can find parameters $a_{i j}, b_{i j}, c_{i j}$, and $d_{i j}$ for $i=0,1,2$ and $j=1, \ldots, 4$ such that system $(10)$ has exactly 0, 1, 2, 3 or 4 limit cycles.

Proposition 3 and 4 are proved in Section 3.

1.6. Example 2. In the following example we solve a problem which is not of type (7).

Let $h(x, y)=y-x^{2}$. The set $\Sigma=h^{-1}(0)$ is a regular manifold which splits the set $\mathbb{R}^{2} \backslash \Sigma$ in two disjoint open regions. We consider the following system

$$
\left(\begin{array}{c}
\dot{x} \\
\dot{y}
\end{array}\right)=\left\{\begin{array}{l}
x+\varepsilon P^{1}(x, y)+\varepsilon^{2} P^{2}(x, y), \\
-y+\varepsilon Q^{1}(x, y)+\varepsilon^{2} Q^{2}(x, y), \\
x+\varepsilon R^{1}(x, y)+\varepsilon^{2} R^{2}(x, y,) \\
-y+\varepsilon S^{1}(x, y)+\varepsilon^{2} S^{2}(x, y),
\end{array} \quad \text { if } \quad h(x, y) \geq 0,\right.
$$


where

$$
\begin{aligned}
& P_{i}=p_{00}^{i}+p_{10}^{i} x+p_{01}^{i} y+p_{20}^{i} x^{2}+p_{11}^{i} x y+p_{02}^{i} y^{2} \\
& Q_{i}=q_{00}^{i}+q_{10}^{i} x+q_{01}^{i} y+q_{20}^{i} x^{2}+q_{11}^{i} x y+q_{02}^{i} y^{2} \\
& R_{i}=r_{00}^{i}+r_{10}^{i} x+r_{01}^{i} y+r_{20}^{i} x^{2}+r_{11}^{i} x y+r_{02}^{i} y^{2} \\
& S_{i}=s_{00}^{i}+s_{10}^{i} x+s_{01}^{i} y+s_{20}^{i} x^{2}+s_{11}^{i} x y+s_{02}^{i} y^{2}
\end{aligned}
$$

for $i=1,2$.

Let $\mathcal{B}$ denote the set of conditions

$$
\begin{aligned}
& p_{00}^{1}=p_{10}^{1}=q_{00}^{1}=q_{01}^{1}=q_{02}^{1}=s_{00}^{1}=s_{02}^{1}=0 \\
& q_{10}^{1}=-p_{01}^{1}-2 p_{20}^{1}, \quad q_{11}^{1}=-p_{02}^{1}-2 p_{20}^{1}, \\
& q_{20}^{1}=-p_{11}^{1}, \quad s_{01}^{1}=-r_{10}^{1}, \quad \text { and } \\
& s_{20}^{1}=3 r_{10}^{1}-r_{11}^{1} .
\end{aligned}
$$

Our results on the limit cycles of system (11) are given in the next two propositions.

Proposition 5. For $|\varepsilon|>0$ sufficiently small and using Theorem A system (11) has at most 4 limit cycles for any chosen of parameters for which the conditions of $\mathcal{B}$ do not hold. Moreover we can find parameters $p_{i j}^{1}, q_{i j}^{1}, r_{i j}^{1}$, and $s_{i j}^{1}$ for $i j=00,10,01,20,11,02$ such that system (11) has exactly 0, 1, 2, 3 or 4 limit cycles.

Proposition 6. For $|\varepsilon|>0$ sufficiently small and using Theorem B system (11) has at most 6 limit cycles for any chosen of parameters for which the conditions of $\mathcal{B}$ hold. Moreover we can find parameters $p_{01}^{1}, p_{20}^{1}, p_{11}^{1}, p_{02}^{1}, s_{10}^{1}, r_{i j}^{1} p_{i j}^{2}, q_{i j}^{2}, r_{i j}^{2}$, and $s_{i j}^{2}$ for $i j=00,10,01,20,11,02$ such that system (11) has exactly $0,1,2,3,4,5$ or 6 limit cycles.

Proposition 5 and 6 are proved in Section 3.

\section{Proofs of Theorems A And B}

In order to prove Theorems A and B we need some preliminary results. As usual $\mu$ denotes the Lebesgue Measure.

Lemma 7. The averaged functions (5) and (6) are continuous in $C$.

Proof. Let $z_{0} \in C$ and let $V$ be a neighborhood of $z_{0}$ with a compact closure contained in $C$. For $z \in V$ we define the sets $A_{z}^{i}(t)=\left\{s \in[0, T]:(s, z) \in S^{i}\right\}$, and $A_{z}^{0}(t)=\{s \in[0, T]:(s, z) \in \Sigma\}$. 
From hypothesis $(H a 2)$ we have that $\mu\left(A_{z}^{0}(t)\right)=0$ for every $t \in[0, T]$. So

$$
\begin{aligned}
\left|y_{1}\left(t, z_{0}\right)-y_{1}(t, z)\right| & =\left|\sum_{j=1}^{M} \int_{A_{z_{0}}^{j}(t)} F_{0}^{j}\left(s, z_{0}\right) d s-\sum_{j=1}^{M} \int_{A_{z}^{j}(t)} F^{j}(s, z) d s\right| \\
& \leq \sum_{j=1}^{M}\left|\int_{A_{z_{0}}^{j}(t)} F_{1}^{j}\left(s, z_{0}\right) d s-\int_{A_{z}^{j}(t)} F^{j}(s, z) d s\right| \\
& \leq \sum_{j=1}^{M} \int_{A_{z_{0}}^{j}(t) \cap A_{z}^{j}(t)} F_{1}^{j}\left(s, z_{0}\right)-F_{1}^{j}(s, z)\left|d s+\sum_{j=1}^{M}\right| \int_{A_{z_{0}}^{j}(t) \backslash A_{z}^{j}(t)} F_{1}^{j}\left(s, z_{A_{z}} F_{1}^{j}(t) \backslash A_{z_{0}}^{j}(t) \mid\right. \\
& \leq M T L\left|z_{0}-z\right|+\sum_{j=1}^{M}\left|\int_{A_{z_{0}}^{j}(t) \backslash A_{z}^{j}(t)} F_{j}^{j}\left(s, z_{0}\right) d s-\int_{A_{z}^{j}(t) \backslash A_{z_{0}}^{j}(t)} F^{j}(s, z) d s\right| \\
& \leq M T L\left|z_{0}-z\right|+\sum_{j=1}^{M} L_{1, j}\left(\mu\left(A_{z_{0}}^{j}(t) \backslash A_{z}^{j}(t)\right)+\mu\left(A_{z}^{j}(t) \backslash A_{z_{0}}^{j}(t)\right)\right),
\end{aligned}
$$

where $L$ is maximum of the Lipschitz constants of the functions $F_{i}^{j}$ for $j=1,2, \ldots, M$, and $L_{1, j}=\max \left\{F_{1}^{j}(s, z):(s, z) \in[0, T] \times \bar{V}\right\}$ for $j=1,2, \ldots, M$. We observe that $\mu\left(A_{z_{0}}^{j}(t) \backslash A_{z}^{j}(t)\right) \rightarrow$ 0 and $\mu\left(A_{z}^{j}(t) \backslash A_{z_{0}}^{j}(t)\right) \rightarrow 0$, as $z \rightarrow z_{0}$ for every $t \in[0, T]$. Thus $\left|y_{1}\left(t, z_{0}\right)-y_{1}(t, z)\right| \rightarrow 0$, as $z \rightarrow z_{0}$ for every $t \in[0, T]$. So the function $y_{1}(t, z)$ is continuous in $C$ for each $t \in[0, T]$. Since $f_{1}(z)=y_{1}(T, z)$, we conclude that the averaged function $f_{1}$ is continuous in $C$.

Given $z_{0} \in C$ and repeating the computations done for $y_{1}$, now for $\int_{0}^{t} F_{2}(s, z) d s$ we get that this function is continuous for $z \in C$. So for proving the continuity of the function $f_{2}$ it is sufficient to estimate the difference

$$
D\left(z_{0}, z\right)=\left|\int_{0}^{T}\left(D_{z} F_{1}\left(t, z_{0}\right) y_{1}\left(t, z_{0}\right)-D_{z} F_{1}(t, z) y_{1}(t, z)\right) d t\right|,
$$

for $z \in V$, being $V$ the above neighborhood of $z_{0}$ with a compact closure. So

$$
\begin{aligned}
D\left(z_{0}, z\right) & \leq \int_{0}^{T}\left|D_{z} F_{1}\left(t, z_{0}\right)-D_{z} F_{1}(t, z)\right|\left|y_{1}\left(t, z_{0}\right)\right| d t+\int_{0}^{T}\left|D_{z} F_{1}(t, z)\right|\left|y_{1}\left(t, z_{0}\right)-y_{1}(t, z)\right| d t \\
& \leq T Y \int_{0}^{T}\left|D_{z} F_{1}\left(t, z_{0}\right)-D_{z} F_{1}(t, z)\right| d t+T L^{\prime} \int_{0}^{T}\left|y_{1}\left(t, z_{0}\right)-y_{1}(t, z)\right| d t,
\end{aligned}
$$

where $Y=\max \left\{\left|y_{1}(s, z)\right|:(s, z) \in[0, T] \times \bar{V}\right\}$ and $L^{\prime}=\max _{j=1}^{M}\left\{\left|D_{z} F_{1}^{j}(s, z)\right|:(s, z) \in\right.$ $[0, T] \times \bar{V}\}$.

Now from the continuity of $y_{1}(t, z)$ and doing similar computations as the ones done for the discontinuous function $D_{z} F_{1}(t, z)$ we conclude that $D\left(z_{0}, z\right) \rightarrow 0$ when $z \rightarrow z_{0}$, which implies the continuity of the averaged function $f_{2}$ in $C$.

Let $g:\left(-\varepsilon_{0}, \varepsilon_{0}\right) \rightarrow \mathbb{R}^{d}$ be a function defined on a small interval $\left(-\varepsilon_{0}, \varepsilon_{0}\right)$. We say that

1. $g(\varepsilon)=\mathcal{O}\left(\varepsilon^{\ell}\right)$ for some positive integer $\ell$ if there exists constants $\varepsilon_{1}>0$ and $k>0$ such that $\|g(\varepsilon)\| \leq k\left|\varepsilon^{\ell}\right|$ for $-\varepsilon_{1}<\varepsilon<\varepsilon_{1}$.

2. $g(\varepsilon)=o\left(\varepsilon^{\ell}\right)$ for some positive integer $\ell$ if

$$
\lim _{\varepsilon \rightarrow 0} \frac{\|g(\varepsilon)\|}{\varepsilon^{\ell}}=0 .
$$


The symbols $\mathcal{O}$ and $o$ are called the Landau's symbols (see for instance [19]).

Lemma 8 (Fundamental lemma). Let $x(\cdot, z, \varepsilon):\left[0, t_{z}\right) \rightarrow \mathbb{R}^{n}$ be the solution of system (3) with $x(0, z, \varepsilon)=z$.

(a) Under the hypotheses of Theorem $A$, the function $\varepsilon \mapsto x(t, z, \varepsilon)$ is differentiable at $\varepsilon=0$. Moreover $x(t, z, \varepsilon)=z+\varepsilon y_{1}(t, z)+\mathcal{O}\left(\varepsilon^{2}\right)$.

(b) Under the hypotheses of Theorem $B$, the function $\varepsilon \mapsto x(t, z, \varepsilon)$ is differentiable at $\varepsilon=0$. Moreover the $x(t, z, \varepsilon)=z+\varepsilon y_{1}(t, z)+\varepsilon^{2} \int_{0}^{t}\left(D_{z} F_{1}(s, z) y_{1}(s, z)+F_{2}(s, z)\right) d s+\mathcal{O}\left(\varepsilon^{3}\right)$.

Proof. From hypothesis (Ha2) for $z \in C$ the function $t \in\left[0, t_{z}\right) \mapsto x(t, z, \varepsilon)$ is continuous and piecewise differentiable. So for $|\varepsilon|>0$ sufficiently small we can assume that

$$
x(t, z, \varepsilon)=\left\{\begin{array}{clc}
x_{1}(t, z, \varepsilon) & \text { if } \quad 0=t_{\varepsilon}^{0} \leq t \leq t_{\varepsilon}^{1}, \\
x_{2}(t, z, \varepsilon) & \text { if } & t_{\varepsilon}^{1} \leq t \leq t_{\varepsilon}^{2}, \\
\vdots & & \\
x_{i}(t, z, \varepsilon) & \text { if } & t_{\varepsilon}^{i-1} \leq t \leq t_{\varepsilon}^{i}, \\
\vdots & & \\
x_{\kappa}(t, z, \varepsilon) & \text { if } & t_{\varepsilon}^{\kappa-1} \leq t \leq t_{\varepsilon}^{\kappa}=t_{z} \leq T,
\end{array}\right.
$$

for which we have the following recurrence

$$
x_{1}(0, z, \varepsilon)=z \quad \text { and } \quad x_{i+1}\left(t_{\varepsilon}^{i}, z, \varepsilon\right)=x_{i}\left(t_{\varepsilon}^{i}, z, \varepsilon\right),
$$

for $i=1,2, \ldots, \kappa-1$.

The functions $x_{i}(t, z, \varepsilon)$ for $i=1,2, \ldots, \kappa-1$ are of class $\mathcal{C}^{1}$ and satisfy the DPDS (4), i.e. there exists a subsequence $\left(j_{i}\right)$ for $i=1, \ldots, \kappa$ such that

$$
\frac{\partial}{\partial t} x_{i}(t, z, \varepsilon)=\varepsilon F_{1}^{j_{i}}\left(t, x_{i}(t, z, \varepsilon)\right)+\varepsilon^{2} F_{2}^{j_{i}}\left(t, x_{i}(t, z, \varepsilon)\right)+\varepsilon^{3} R^{j_{i}}\left(t, x_{i}(t, z, \varepsilon), \varepsilon\right) .
$$

The function $x_{i}(t, z, \varepsilon)$ is the solution of the Cauchy Problem defined by the differential system (13) together with the initial condition (12), i.e. $x_{i}\left(t_{\varepsilon}^{i-1}, z, \varepsilon\right)=z$ for $i=1$, and $x_{i}\left(t_{\varepsilon}^{i-1}, z, \varepsilon\right)=x_{i-1}\left(t_{\varepsilon}^{i-1}, z, \varepsilon\right)$ for $i=2,3, \ldots, \kappa-1$.

On the other hand, for $i=1,2, \ldots, \kappa-1$ and for each $z \in D$, there exists $\varepsilon_{i}>0$ such that if $\varepsilon \in\left[-\varepsilon_{i}, \varepsilon_{i}\right]$ then the solution $x_{i}(t, z, \varepsilon)$ of $(13)$ is defined in $[0, T]$. Indeed, by the Existence and Uniqueness Theorem of solutions (see, for example, Theorem 1.2.4 of [19]), for $z \in D, x_{i}(t, z, \varepsilon)$ is defined for all $0 \leq t \leq \inf \left(T, d / M_{i}(\varepsilon)\right)$, where

$$
M_{i}(\varepsilon) \geq\left|\varepsilon F_{1}^{j_{i}}\left(t, x_{i}(t, z, \varepsilon)\right)+\varepsilon^{2} F_{2}^{j_{i}}\left(t, x_{i}(t, z, \varepsilon)\right)+\varepsilon^{3} R^{j_{i}}\left(t, x_{i}(t, z, \varepsilon), \varepsilon\right)\right|
$$

for all $t \in[0, T]$, for each $x$ with $|x-z| \leq d$ and for every $z \in \bar{D}$. When $\varepsilon$ is sufficiently small we can take $d / M_{i}(\varepsilon)$ sufficiently large in order that $\inf \left(T, d / M_{i}(\varepsilon)\right)=T$ for all $z \in \bar{V}$. So for any $z \in C$ we have that the solution $x(t, z, \varepsilon)$ of system $(3)$ is also defined for every $t \in[0, T]$, i.e. $t_{z}=T$.

From the continuity of the solution $x(t, z, \varepsilon)$ and by compactness of the set $[0, T] \times \bar{C} \times$ $\left[-\varepsilon_{1}, \varepsilon_{1}\right]$, there exits a compact subset $K$ of $D$ such that $x(t, z, \varepsilon) \in K$ for all $t \in[0, T], z \in \bar{C}$ and $\varepsilon \in\left[-\varepsilon_{1}, \varepsilon_{1}\right]$. Now, by the piecewise continuity of the function $R,|R(s, x(s, z, \varepsilon), \varepsilon)| \leq$ $\max \left\{|R(t, x, \varepsilon)|,(t, x, \varepsilon) \in[0, T] \times K \times\left[-\varepsilon_{1}, \varepsilon_{1}\right]\right\}=N$. Then

$$
\left|\int_{0}^{t} R(s, x(s, z, \varepsilon), \varepsilon) d s\right| \leq \int_{0}^{T}|R(s, x(s, z, \varepsilon), \varepsilon)| d s=T N,
$$


which implies that

$$
\int_{0}^{t} R(s, x(s, z, \varepsilon), \varepsilon) d s=\mathcal{O}(1)
$$

Now for a given $t \in(0, T)$ there exists $\bar{\kappa} \in\{1,2, \ldots, \kappa-1\}$ such that $t \in\left[t_{\varepsilon}^{\bar{\kappa}-1}, t_{\varepsilon}^{\bar{\kappa}}\right)$ and

$$
\begin{aligned}
x(t, z, \varepsilon) & =x_{\bar{\kappa}}(t, z, \varepsilon) \\
& =x_{\bar{\kappa}-1}\left(t_{\varepsilon}^{\bar{\kappa}-1}, z, \varepsilon\right)+\varepsilon \int_{t_{\varepsilon}^{\bar{\kappa}-1}}^{t} F_{1}(s, x(s, z, \varepsilon)) d s+\varepsilon^{2} \int_{t_{\varepsilon}^{\bar{\kappa}-1}}^{t} F_{2}(s, x(s, z, \varepsilon)) d s+\mathcal{O}\left(\varepsilon^{3}\right) .
\end{aligned}
$$

Since

$$
x_{i+1}\left(t_{\varepsilon}^{i+1}, z, \varepsilon\right)=x_{i}\left(t_{\varepsilon}^{i}, z, \varepsilon\right)+\varepsilon \int_{t_{\varepsilon}^{i}}^{t_{\varepsilon}^{i+1}} F_{1}(t, x(t, z, \varepsilon)) d t+\varepsilon^{2} \int_{t_{\varepsilon}^{i}}^{t_{\varepsilon}^{i+1}} F_{2}(t, x(t, z, \varepsilon)) d t+\mathcal{O}\left(\varepsilon^{3}\right),
$$

proceeding by induction on $i$ we obtain

$$
x(t, z, \varepsilon)=z+\varepsilon \int_{0}^{t} F_{1}(s, x(s, z, \varepsilon)) d s+\varepsilon^{2} \int_{0}^{t} F_{2}(s, x(s, z, \varepsilon)) d s+\mathcal{O}\left(\varepsilon^{3}\right) .
$$

Thus let $t^{i}=\lim _{\varepsilon \rightarrow 0} t_{\varepsilon}^{i}$ for $i=1,2, \ldots, \kappa-1$. We compute

$$
\begin{aligned}
\int_{0}^{t} F_{1}(s, x(s, z, \varepsilon)) d s & =\left(\sum_{i=1}^{\bar{\kappa}-1} \int_{t_{\varepsilon}^{i-1}}^{t_{\varepsilon}^{i}} F_{1}^{j_{i}}\left(s, x_{i}(s, z, \varepsilon)\right) d s\right)+\int_{t_{\varepsilon}^{\bar{\kappa}-1}}^{t} F_{1}^{j_{\bar{\kappa}}}\left(s, x_{\bar{\kappa}}(s, z, \varepsilon)\right) d s \\
& =\left(\sum_{i=1}^{\bar{\kappa}-1} \int_{t^{i-1}}^{t^{i}} F_{1}^{j_{i}}\left(s, x_{i}(s, z, \varepsilon)\right) d s\right)+\int_{t^{\bar{\kappa}-1}}^{t} F_{1}^{j_{\bar{\kappa}}}\left(s, x_{\bar{\kappa}}(s, z, \varepsilon)\right) d s+E(\varepsilon),
\end{aligned}
$$

where

$$
\begin{aligned}
E(\varepsilon)= & \sum_{i=1}^{\bar{\kappa}-1}\left(\int_{t_{\varepsilon}^{i-1}}^{t^{i-1}} F_{1}^{j_{i}}\left(s, x_{i}(s, z, \varepsilon)\right) d s-\int_{t_{\varepsilon}^{i}}^{t^{i}} F_{1}^{j_{i}}\left(s, x_{i}(s, z, \varepsilon)\right) d s\right) \\
& +\int_{t_{\varepsilon}^{\bar{\kappa}-1}}^{t^{\bar{\kappa}-1}} F_{1}^{j_{\bar{\kappa}}}\left(s, x_{\bar{\kappa}}(s, z, \varepsilon)\right) d s .
\end{aligned}
$$

It is easy to see that there exists a constant $\tilde{E}$ such that

$$
|E(\varepsilon)| \leq \tilde{E} \sum_{i=0}^{\bar{\kappa}-1}\left|t^{i}-t_{\varepsilon}^{i}\right|
$$

Claim 1. Statement (a) of Lemma 8 holds.

Denoting $t_{\varepsilon}^{i}=t^{i}(\varepsilon)$ we shall prove that $t^{i}(\varepsilon)$ is a $\mathcal{C}^{k}$ function with $k \geq 1$.

From hypothesis $(\mathrm{Ha} 2)$ the solution $x(t, z, 0)=z$ reaches the discontinuity set only on $\Sigma^{c}$, i.e. $\left(t^{i}, z\right) \in \Sigma^{c}$ for every $i=1,2, \ldots, \kappa-1$. Particularly $\left(t^{i}, z\right)$ is a generic point of $\Sigma$. So from the hypothesis $(H a 1)$ there exists a neighbourhood $G_{\left(t^{i}, z\right)}$ of $\left(t^{i}, z\right)$ such that $\mathcal{S}_{\left(t^{i}, z\right)}=G_{\left(t^{i}, z\right)} \cap \Sigma$ is a $\mathcal{C}^{k}$ embedded hypersurface of $\mathbb{R}^{d+1}$ with $k \geq 1$. So it is well known that $\mathcal{S}_{\left(t^{i}, z\right)}$ can be locally described as the inverse image of a regular value of a $\mathcal{C}^{k}$ function. Thus we can find a $\mathcal{C}^{k}$ function $h_{i}: G_{\left(t^{i}, z\right)} \rightarrow \mathbb{R}$ such that $\tilde{G}_{\left(t^{i}, z\right)} \cap \mathcal{S}_{\left(t^{i}, z\right)}=h_{i}^{-1}(0) \cap \Sigma$. Here $\tilde{G}_{\left(t^{i}, z\right)}$ is an open subset such that $\left(t^{i}, z\right) \in \tilde{G}_{\left(t^{i}, z\right)} \subseteq G_{\left(t^{i}, z\right)}$. 
For $(t, z) \in \tilde{G}_{\left(t^{i}, z\right)}$ system (4) can be written as the autonomous system

$$
\left(\begin{array}{c}
\tau^{\prime} \\
x^{\prime}
\end{array}\right)=\left\{\begin{array}{lll}
X(\tau, x, \varepsilon) & \text { if } \quad h_{i}(\tau, x)>0 \\
Y(\tau, x, \varepsilon) & \text { if } \quad h_{i}(\tau, x)<0
\end{array}\right.
$$

where

$$
\begin{gathered}
X(\tau, x, \varepsilon)=\left(\begin{array}{c}
1 \\
\varepsilon F_{1}^{j_{i+1}}(\tau, x)+\varepsilon^{2} F_{2}^{j_{i+1}}(\tau, x)+\varepsilon^{3} R^{j_{i+1}}(\tau, x, \varepsilon)
\end{array}\right), \\
Y(\tau, x, \varepsilon)=\left(\begin{array}{c}
1 \\
\varepsilon F_{1}^{j_{i}}(\tau, x)+\varepsilon^{2} F_{2}^{j_{i}}(\tau, x)+\varepsilon^{3} R^{j_{i}}(\tau, x, \varepsilon)
\end{array}\right) .
\end{gathered}
$$

Now we define $H_{i}(t, \varepsilon)=h_{i}\left(t, x_{i}(t, z, \varepsilon)\right)$. We have that $H_{i}\left(t^{i}, 0\right)=0$. Moreover, from hypothesis (Ha2) we have that $X h_{i}\left(t^{i}, z, \varepsilon\right) Y h_{i}\left(t^{i}, z, \varepsilon\right)>0$. Since $X h_{i}\left(t^{i}, z, 0\right) Y h_{i}\left(t^{i}, z, 0\right)>0$ is equal to

$$
\left\langle\left(\frac{\partial}{\partial t} h_{i}\left(t^{i}, z\right), \frac{\partial}{\partial z} h_{i}\left(t^{i}, z\right)\right),(1,0)\right\rangle\left\langle\left(\frac{\partial}{\partial t} h_{i}\left(t^{i}, z\right), \frac{\partial}{\partial z} h_{i}\left(t^{i}, z\right)\right),(1,0)\right\rangle>0 .
$$

So we conclude that $\left((\partial / \partial t) h_{i}\left(t^{i}, z\right)\right)^{2}>0$. Thus

$$
\begin{aligned}
\frac{\partial}{\partial t} H_{i}\left(t^{i}, 0\right) & =\left.\frac{\partial}{\partial t} h_{i}\left(t, x_{i}(t, z, \varepsilon)\right)\right|_{\left(t^{i}, 0\right)} \\
& =\frac{\partial}{\partial t} h_{i}\left(t^{i}, x_{i}\left(t^{i}, z, 0\right)\right)+\frac{\partial}{\partial z} h_{i}\left(t^{i}, x_{i}\left(t^{i}, z, 0\right)\right) \frac{\partial}{\partial t} x_{i}\left(t^{i}, z, 0\right) \\
& =\frac{\partial}{\partial t} h_{i}\left(t^{i}, x_{i}\left(t^{i}, z, 0\right)\right) \neq 0
\end{aligned}
$$

because from $(13)(\partial / \partial t) x_{i}(t, z, 0)=0$. Hence from the Implicit Function Theorem, $t^{i}(\varepsilon)$ is a $\mathcal{C}^{k}$ with $H\left(t^{i}(\varepsilon), \varepsilon\right)=0$ for every $|\varepsilon|>0$ sufficiently small and $t^{i}(0)=t^{i}$. So $t^{i}(\varepsilon)=$ $t^{i}+\left(t^{i}\right)^{\prime}(0) \varepsilon+o(\varepsilon)$ for every $i=1,2, \ldots, \kappa-1$, because $k \geq 1$. This implies that $E(\varepsilon)=\mathcal{O}(\varepsilon)$.

Since $F_{1}^{j_{i}}$ is Lipschitz for $i=1,2, \ldots, \kappa$ in the variable $x$, we have that

$$
\begin{aligned}
& \left|\int_{t^{i-1}}^{t^{i}} F_{1}^{j_{i}}\left(s, x_{i}(s, z, \varepsilon)\right) d s-\int_{t^{i-1}}^{t^{i}} F_{1}^{j_{i}}(s, z) d s\right| \leq \int_{t^{i-1}}^{t^{i}}\left|F_{1}^{j_{i}}\left(s, x_{i}(s, z, \varepsilon)\right)-F_{1}^{j_{i}}(s, z)\right| d s \\
& \leq L_{j_{i}} \int_{t^{i-1}}^{t^{i}}\left|x_{i}(s, z, \varepsilon)-z\right| d s \leq L_{j_{i}} T\left|x_{i}(s, z, \varepsilon)-z\right|=\mathcal{O}(\varepsilon),
\end{aligned}
$$

where $L_{j_{i}}$ is the Lipschitz constant of the function $F_{1}^{j_{i}}$. This implies that

$$
\int_{t^{i-1}}^{t^{i}} F_{1}^{j_{i}}\left(s, x_{i}(s, z, \varepsilon)\right) d s=\int_{t^{i-1}}^{t^{i}} F_{1}^{j_{i}}(s, z) d s+\mathcal{O}(\varepsilon)
$$

for every $i=1,2, \ldots, \kappa$. Going back to the inequality (16) we have

$$
\begin{aligned}
\int_{0}^{t} F_{1}(s, x(s, z, \varepsilon)) d s & =\left(\sum_{i=1}^{\bar{\kappa}-1} \int_{t^{i-1}}^{t^{i}} F_{1}^{j_{i}}(s, z) d s\right)+\int_{t^{\bar{\kappa}-1}}^{t} F_{1}^{j_{\bar{\kappa}}}(s, z) d s+E(\varepsilon)+\mathcal{O}(\varepsilon) \\
& =\int_{0}^{t} F_{1}(s, z) d s+\mathcal{O}(\varepsilon) .
\end{aligned}
$$


Hence from (15) and (19) we get that

$$
x(t, z, \varepsilon)=z+\varepsilon \int_{0}^{t} F_{1}(s, z) d s+\mathcal{O}\left(\varepsilon^{2}\right)
$$

Therefore the claim 1 is proved.

Claim 2. Statement (b) of Lemma 8 holds.

First we shall prove that $E(\varepsilon)=\mathcal{O}\left(\varepsilon^{2}\right)$. From hypothesis $(H a 2)$ and following the steps of the proof of Claim 1 we can find a $\mathcal{C}^{k}$ function $h_{i}: G_{\left(t^{i}, z\right)} \rightarrow \mathbb{R}$, now with $k \geq 2$, such that $\tilde{G}_{\left(t^{i}, z\right)} \cap \mathcal{S}_{\left(t^{i}, z\right)}=h_{i}^{-1}(0) \cap \Sigma$. Again, $\tilde{G}_{\left(t^{i}, z\right)}$ is an open subset such that $\left(t^{i}, z\right) \in \tilde{G}_{\left(t^{i}, z\right)} \subseteq G_{\left(t^{i}, z\right)}$. Applying the Inverse Function Theorem we conclude that $t^{i}(\varepsilon)$ is a $\mathcal{C}^{2}$ function. So

$$
t^{i}(\varepsilon)=t^{i}+\left(t^{i}\right)^{\prime}(0) \varepsilon+\mathcal{O}\left(\varepsilon^{2}\right)
$$

From statement $(a)$ and (21) the function $\left.\varepsilon \mapsto x\left(t^{i}(\varepsilon), z, \varepsilon\right)\right)$ is differentiable at $\varepsilon=0$. Moreover $y_{1}(t, z)=(\partial / \partial \varepsilon) x(t, z, 0)$. Since for $|\varepsilon|>0$ sufficiently small $h_{i}\left(t^{i}(\varepsilon), x\left(t^{i}(\varepsilon), z, \varepsilon\right)\right)=$ 0 , so

$$
\begin{aligned}
0 & =\frac{\partial}{\partial \varepsilon} h\left(t^{i}(\varepsilon), x\left(t^{i}(\varepsilon), z, \varepsilon\right)\right) \\
& =\frac{\partial}{\partial t} h\left(t^{i}, z\right)\left(t^{i}\right)^{\prime}(0)+\frac{\partial}{\partial z} h\left(t^{i}, z\right)\left(\frac{\partial}{\partial t} x\left(t^{i}, z, 0\right)\left(t^{i}\right)^{\prime}(0)+\left.\frac{\partial}{\partial \varepsilon} x\left(t^{i}, z, \varepsilon\right)\right|_{\varepsilon=0}\right) \\
& =\left\langle\nabla h\left(t^{i}, z\right),\left(\left(t^{i}\right)^{\prime}(0), y_{1}\left(t^{i}, z\right)\right)\right\rangle
\end{aligned}
$$

because $(\partial / \partial t) x(t, z, 0)$ is the vector field (4) for $\varepsilon=0$. So $\left(\left(t^{i}\right)^{\prime}(0), y_{1}\left(t^{i}, z\right)\right) \in T_{\left(t^{i}, z\right)} \Sigma$. From hypothesis $(H b 2)$ we conclude that $\left(t^{i}\right)^{\prime}(0)=0$. From (17) and (21) it follows that $E(\varepsilon)=\mathcal{O}\left(\varepsilon^{2}\right)$.

Now for $t_{i-1} \leq t \leq t^{i}$ we prove that

$$
\left|F_{1}^{j_{i}}(t, x(t, z, \varepsilon))-F_{1}^{j_{i}}(t, z)-\varepsilon D_{z} F_{1}^{j_{i}}(t, z) y_{1}(t, z)\right|=\mathcal{O}\left(\varepsilon^{2}\right)
$$

For this goal we define

$$
G_{i}(\lambda)=F_{1}^{j_{i}}\left(t, \lambda x_{i}(t, z, \varepsilon)+(1-\lambda) z\right) .
$$

Computing the derivative in $\lambda$ we get

$$
G^{\prime}(\lambda)=D_{z} F_{1}^{j_{i}}\left(t, \lambda x_{i}(t, z, \varepsilon)+(1-\lambda) z\right)\left(x_{i}(t, z, \varepsilon)-z\right)
$$

So from the Fundamental Theorem of Calculus and observing that for $t_{i-1} \leq t \leq t^{i}, x_{i}(t, z, \varepsilon)=$ $x(t, z, \varepsilon)$ it follows that

$$
G(1)-G(0)=\int_{0}^{1} D_{z} F_{1}^{j_{i}}(t, \lambda x(t, z, \varepsilon)+(1-\lambda) z)(x(t, z, \varepsilon)-z) d \lambda
$$


Thus

$$
\begin{aligned}
& \frac{1}{\varepsilon^{2}}\left(F_{1}^{j_{i}}\left(t, x_{i}(t, z, \varepsilon)\right)-F_{1}^{j_{i}}(t, z)-\varepsilon D_{z} F_{1}^{j_{i}}(t, z) y_{1}(t, z)\right)= \\
& \frac{1}{\varepsilon^{2}}\left(G(1)-G(0)-\varepsilon D_{z} F_{1}^{j_{i}}(t, z) y_{1}(t, z)\right)= \\
& \frac{1}{\varepsilon} \int_{0}^{1} D_{z} F_{1}^{j_{i}}(t, \lambda x(t, z, \varepsilon)+(1-\lambda) z) \frac{(x(t, z, \varepsilon)-z)}{\varepsilon} d \lambda-\frac{1}{\varepsilon} D_{z} F_{1}^{j_{i}}(t, z) y_{1}(t, z)= \\
& \frac{1}{\varepsilon}\left(\int_{0}^{1} D_{z} F_{1}^{j_{i}}(t, \lambda x(t, z, \varepsilon)+(1-\lambda) z) d \lambda\right) \int_{0}^{t} F_{1}(s, x(s, z, \varepsilon)) d s- \\
& \frac{1}{\varepsilon} D_{z} F_{1}^{j_{i}}(t, z) y_{1}(t, z)+\mathcal{O}(1)= \\
& \frac{1}{\varepsilon}\left(\int_{0}^{1}\left[D_{z} F_{1}^{j_{i}}(t, \lambda x(t, z, \varepsilon)+(1-\lambda) z)-D_{z} F_{1}^{j_{i}}(t, z)\right] d \lambda\right) \int_{0}^{t} F_{1}(s, x(s, z, \varepsilon)) d s+ \\
& \frac{1}{\varepsilon} D_{z} F_{1}^{j_{i}}(t, z)\left[\int_{0}^{t} F_{1}(s, x(s, z, \varepsilon))-F_{1}(s, z) d s\right]+\mathcal{O}(1) .
\end{aligned}
$$

Let $B_{i}=\max \left\{\left|F_{1}^{j_{i}}\left(s, x_{i}(s, z, \varepsilon)\right)\right|:(t, z) \in[0, T] \times \bar{C}\right\}$. Observing that $F_{1}^{j_{i}}$ is locally Lipschitz in the variable $z$, and (from (19)) that $\int_{0}^{t} F_{1}(s, x(s, z, \varepsilon))-\int_{0}^{t} F_{1}(s, z)=\mathcal{O}(\varepsilon)$, it follows that

$$
\begin{aligned}
& \left|\frac{1}{\varepsilon^{2}}\left(F_{1}^{j_{i}}\left(t, x_{i}(t, z, \varepsilon)\right)-F_{1}^{j_{i}}(t, z)-\varepsilon D_{z} F_{1}^{j_{i}}(t, z) y_{1}(t, z)\right)\right| \leq \\
& \frac{1}{\varepsilon} \int_{0}^{1}\left|D_{z} F_{1}^{j_{i}}(t, \lambda x(t, z, \varepsilon)+(1-\lambda) z)-D_{z} F_{1}^{j_{i}}(t, z)\right| d \lambda\left|\int_{0}^{t} F_{1}(s, x(s, z, \varepsilon)) d s\right|+ \\
& \frac{1}{\varepsilon}\left|D_{z} F_{1}^{j_{i}}(t, z)\right|\left|\int_{0}^{t} F_{1}(s, x(s, z, \varepsilon))-F_{1}(s, z) d s\right|+\mathcal{O}(1) \leq \\
& T L_{i} B_{i} \frac{|x(t, z, \varepsilon)-z|}{\varepsilon}+\mathcal{O}(1)=\mathcal{O}(1),
\end{aligned}
$$

where $L_{i}$ is the Lipschitz constant of the function $D_{z} F_{1}^{j_{i}}$. Hence for $t^{i-1} \leq t \leq t^{i}$ and for every $i=1,2, \ldots, \kappa$ the equality $(22)$ holds which implies that $F_{1}^{j_{i}}(t, x(t, z, \varepsilon))=F_{1}^{j_{i}}(t, z)+$ $\varepsilon D_{z} F_{1}^{j_{i}}(t, z) y_{1}(t, z)+\mathcal{O}\left(\varepsilon^{2}\right)$. So

$$
\int_{t^{i-1}}^{t} F_{1}^{j_{i}}\left(s, x_{i}(s, z, \varepsilon)\right) d s=\int_{t^{i-1}}^{t} F_{1}^{j_{i}}(s, z) d s-\varepsilon \int_{t^{i-1}}^{t} D_{z} F_{1}^{j_{i}}(s, z) y_{1}(s, z) d s+\mathcal{O}\left(\varepsilon^{2}\right) .
$$

Going back to the inequality (16) we have

$$
\begin{aligned}
\int_{0}^{t} F_{1}(s, x(s, z, \varepsilon)) d s= & \left(\sum_{i=1}^{\bar{\kappa}-1} \int_{t^{i-1}}^{t^{i}} F_{1}^{j_{i}}(s, z) d s\right)+\int_{t^{\bar{\kappa}-1}}^{t} F_{1}^{j_{\bar{\kappa}}}(s, z) d s \\
& -\varepsilon\left(\sum_{i=1}^{\bar{\kappa}-1} \int_{t^{i-1}}^{t^{i}} D_{z} F_{1}^{j_{i}}(s, z) y_{1}(s, z) d s-\int_{t^{\bar{\kappa}-1}}^{t} D_{z} F_{1}^{j_{\bar{\kappa}}}(s, z) y_{1}(s, z) d s\right) \\
& +\mathcal{O}\left(\varepsilon^{2}\right)+E(\varepsilon) \\
& =\int_{0}^{t} F_{1}(s, z) d s-\varepsilon \int_{0}^{t} D_{z} F_{1}(s, z) y_{1}(s, z) d s+\mathcal{O}\left(\varepsilon^{2}\right) .
\end{aligned}
$$


Analogously to the proof of statement $(a)$ and using that $E(\varepsilon)=\mathcal{O}\left(\varepsilon^{2}\right) \subset \mathcal{O}(\varepsilon)$ we can show that

$$
\int_{0}^{t} F_{2}(s, x(s, z, \varepsilon)) d s=\int_{0}^{t} F_{2}(s, z) d s+\mathcal{O}(\varepsilon) .
$$

So from (15), (23) and (24) we get

$$
x(t, z, \varepsilon)=z+\varepsilon \int_{0}^{t} F_{1}(s, z) d s-\varepsilon^{2} \int_{0}^{t}\left(D_{z} F_{1}(s, z) y_{1}(s, z)+F_{2}(s, z)\right) d s+\mathcal{O}\left(\varepsilon^{3}\right),
$$

This completes the proof of the claim 2 .

Proof of Theorem $A$. Let $f$ be the function such that $\varepsilon f(z, \varepsilon)=x(T, z, \varepsilon)-z$. From statement (a) of Lemma 8 we have that

$$
f(z, \varepsilon)=f_{1}(z)+\mathcal{O}(\varepsilon),
$$

where the function $f_{1}$ is the one defined in (5). Clearly $x(t, z, \varepsilon)$ is a $T$-periodic solution if and only if $f(z, \varepsilon)=0$, because $x(t, z, \varepsilon)$ is defined for all $t \in[0, T]$.

From the Brouwer degree theory (see Proposition 12 of the appendix) and hypothesis ( $\mathrm{Ha} 3$ ) we have for $|\varepsilon|>0$ sufficiently small that

$$
d_{B}\left(f_{1}(z), U, a\right)=d_{B}(f(z, \varepsilon), U, a) \neq 0 .
$$

Hence, by item (i) of Theorem 10 (see the Appendix), $0 \in f(U, \varepsilon)$ for $|\varepsilon|>0$ sufficiently small, i.e, there exists $a_{\varepsilon} \in U$ such that $f\left(a_{\varepsilon}, \varepsilon\right)=0$. Therefore, for $|\varepsilon|>0$ sufficiently small, $x\left(t, a_{\varepsilon}, \varepsilon\right)$ is a periodic solution of (3). We can choose $a_{\varepsilon}$ such that $a_{\varepsilon} \rightarrow a$ when $\varepsilon \rightarrow 0$, because $f(z, \varepsilon) \neq 0$ in $U \backslash\{a\}$. This completes the proof of the theorem.

Proof of Theorem B. Now let $f$ be a function such that $\varepsilon^{2} f(z, \varepsilon)=x(T, z, \varepsilon)-z$. From statement (b) of Lemma 8 we have that

$$
f(z, \varepsilon)=f_{2}(z)+\mathcal{O}(\varepsilon)
$$

where the function $f_{2}$ is the one defined in (6). From here the proof follows in a similar way to the proof of Theorem A.

\section{Proofs of Propositions 3, 4, 5 And 6}

Proof of Proposition 3. The linear DPDS (10) in polar coordinates $(r, \theta)$ becomes

$$
\begin{aligned}
\dot{r}= & \varepsilon\left(a_{0 i} \cos \theta+a_{1 i} r \cos ^{2} \theta+b_{0 i} \sin \theta+a_{2 i} r \cos \theta \sin \theta+b_{1 i} r \cos \theta \sin \theta+b_{2 i} r \sin ^{2} \theta\right)+ \\
& \varepsilon^{2}\left(c_{0 i} \cos \theta+c_{1 i} r \cos ^{2} \theta+c_{2 i} r \cos \theta \sin \theta+d_{1 i} r \cos \theta \sin \theta+d_{2 i} r \sin ^{2} \theta+d_{0 i} \sin \theta\right), \\
\dot{\theta}= & -1-\frac{\varepsilon}{r}\left(-b_{0 i} \cos \theta-b_{1 i} r \cos ^{2} \theta+a_{0 i} \sin \theta+a_{1 i} r \cos \theta \sin \theta-b_{2 i} r \cos \theta \sin \theta+a_{2 i} r \sin ^{2} \theta\right)- \\
& \frac{\varepsilon^{2}}{r}\left(-d_{0 i} \cos \theta-d_{1 i} r \cos ^{2} \theta+c_{0 i} \sin \theta+c_{1 i} r \cos \theta \sin \theta-d_{2 i} r \cos \theta \sin \theta+c_{2 i} r \sin ^{2} \theta\right),
\end{aligned}
$$

with $i=1$ if $0<\theta<\pi / 2, i=2$ if $\pi / 2<\theta<\pi, i=3$ if $\pi<\theta<3 \pi / 2$ and $i=4$ if $3 \pi / 2<\theta<2 \pi$. Taking the angle $\theta$ as the new independent variable the DPDS (10) becomes

$$
\dot{r}=\varepsilon F_{1 i}+\varepsilon^{2} F_{2 i}+\mathcal{O}\left(\varepsilon^{3}\right),
$$


where

$$
\begin{aligned}
F_{1 i}= & -r\left(a_{0 i} \cos \theta+a_{1 i} r \cos ^{2} \theta+b_{0 i} \sin \theta+a_{2 i} r \cos \theta \sin \theta+b_{1 i} r \cos \theta \sin \theta+b_{2 i} r \sin ^{2} \theta\right), \\
F_{2 i}= & \frac{1}{r}\left(-b_{1 i} r \cos ^{2} \theta-b_{0 i} \cos \theta+a_{1 i} r \sin \theta \cos \theta-b_{2 i} r \sin \theta \cos \theta+a_{2 i} r \sin ^{2} \theta+a_{0 i} \sin \theta\right) \\
& \left(a_{1 i} r \cos ^{2} \theta+a_{0 i} \cos \theta+a_{2 i} r \sin \theta \cos \theta+b_{1 i} r \sin \theta \cos \theta+b_{2 i} r \sin ^{2} \theta+b_{0 i} \sin \theta\right) \\
& -\left(c_{1 i} r \cos ^{2} \theta+c_{0 i} \cos \theta+c_{2 i} r \sin \theta \cos \theta+d_{1 i} r \sin \theta \cos \theta+d_{2 i} r \sin ^{2} \theta+d_{0 i} \sin \theta\right) .
\end{aligned}
$$

By Proposition 3 the DPDS (25) is under the assumptions of Theorem A. Computing the averaged function $f_{1}$ we obtain

$$
\begin{aligned}
f_{1}(r)= & \frac{1}{4} r\left(-4 a_{01}+4\left(a_{02}+a_{03}-a_{04}-b_{01}-b_{02}+b_{03}+b_{04}\right)\right. \\
& -\left(2 a_{21}-2\left(a_{22}-a_{23}+a_{24}-b_{11}+b_{12}-b_{13}+b_{14}\right)\right. \\
& \left.\left.+\left(a_{11}+a_{12}+a_{13}+a_{14}+b_{21}+b_{22}+b_{23}+b_{24}\right) \pi\right) r\right) .
\end{aligned}
$$

Clearly $f_{1}$ has at most 1 zero. Moreover we can choose the coefficients $a_{i j}, i=0,1, j=1, \ldots, 4$ in such a way that $f_{1}$ will have a simple positive zero. Hence the proposition is proved.

Proof of Proposition 4. We choose the coefficients $a_{i j}, i=0,1, j=1, \ldots, 4$ such that the conditions $\mathcal{A}$ hold. Then $f_{1}(r) \equiv 0$. From Proposition 2 the DPDS (25) is under the assumptions of Theorem B. Using some algebraic manipulator as Mathematica or Maple we obtain

$$
f_{2}(r)=k_{0}+k_{1} r+k_{2} r^{2}+k_{3} r^{3}+k_{4} r^{4}
$$

where $k_{i}, i=0, \ldots, 4$ depends freely on the coefficients $a_{i j}, i=0,1, j=1, \ldots, 4$. Function (27) is a polynomial in the variable $r$ of degree 4 . Clearly function $f_{2}$ has at most 4 zeros. Moreover we can choose coefficients $a_{i j}, i=0,1, j=1, \ldots, 4$ such that (27) has $0,1,2,3$ or 4 simple zeros. So Proposition 4 is proved.

In order to prove the Propositions 5 and 6 we have to introduce the concept of ECT-systems and prove one lemma.

Let $I$ be a proper real interval of $\mathbb{R}$. We say that an ordered set of complex-valued functions $F=\left(f_{0}, f_{1}, \ldots, f_{n}\right)$ defined on $I$ is an Extended Chebyshev system or ET-system on $I$ if and only if any nontrivial linear combination of the functions of $F$ has at most $n$ zeros counting multiplicities. We say that $F$ is an Extended Complete Chebyshev system or an ECT-system on $I$ if and only if for any $k, 0 \leq k \leq n,\left(f_{0}, f_{1}, \ldots, f_{k}\right)$ is an ET-system. For more details, see the book of Karlin and Studden [14].

In order to prove that $F$ is a ECT-system on $I$ is sufficient and necessary to show that $W\left(f_{0}, f_{1}, \ldots, f_{k}\right)(t) \neq 0$ on $I$ for $0 \leq K \leq n$. Here $W\left(f_{0}, f_{1}, \ldots, f_{k}\right)(t)$ denotes the Wronskians of the functions $\left(f_{0}, f_{1}, \ldots, f_{k}\right)$ with respect to $t$. We recall the definition of the Wronskian.

$$
W\left(f_{0}, f_{1}, \ldots, f_{k}\right)(t)=\left|\begin{array}{cccc}
f_{0}(t) & f_{1}(t) & \cdots & f_{k}(t) \\
f_{0}^{\prime}(t) & f_{1}^{\prime}(t) & \cdots & f_{k}^{\prime}(t) \\
\vdots & \vdots & \ddots & \vdots \\
f_{0}^{(k)}(t) & f_{1}^{(k)}(t) & \cdots & f_{k}^{(k)}(t)
\end{array}\right|
$$

We also recall the Descartes Theorem about the number of zeros of a real polynomial (for a proof see for instance either the pages 82 and 83 of [4], or the appendix of [17]). 
Descartes Theorem Consider the real polynomial $p(x)=a_{i_{1}} x^{i_{1}}+a_{i_{2}} x^{i_{2}}+\cdots+a_{i_{r}} x^{i_{r}}$ with $0 \leq i_{1}<i_{2}<\cdots<i_{r}$ and $a_{i_{j}} \neq 0$ real constants for $j \in\{1,2, \cdots, r\}$. When $a_{i_{j}} a_{i_{j+1}}<0$, we say that $a_{i_{j}}$ and $a_{i_{j+1}}$ have a variation of sign. If the number of variations of signs is $m$, then $p(x)$ has at most $m$ positive real roots.

Now consider the functions

$$
\begin{aligned}
g_{1}(u) & =1, \\
g_{2}(u) & =u^{2}, \\
g_{3}(u) & =u^{4}, \\
g_{4}(u) & =u\left(2+u^{2}\right) \arccos \left(\frac{u}{\sqrt{2+u^{2}}}\right), \\
g_{5}^{1}(u) & =u\left(2+u^{2}\right), \\
g_{5}^{2}(u) & =u\left(2+u^{2}\right)\left(\pi-\arccos \left(\frac{u}{\sqrt{2+u^{2}}}\right)\right), \\
g_{6}(u) & =\sqrt{2} u^{6}-u\left(8-4 u^{4}-u^{6}\right)\left(\frac{\pi}{2}+\arcsin \left(\frac{u}{\sqrt{2+u^{2}}}\right)\right), \quad \text { and } \\
g_{7}(u) & =-\sqrt{2} u^{6}-\frac{3 \pi u^{3}\left(2+u^{2}\right)^{2}}{2}-u\left(8-4 u^{4}-u^{6}\right) \arccos \left(\frac{u}{\sqrt{2+u^{2}}}\right) .
\end{aligned}
$$

We define the sets of functions $G^{1}=\left\{g_{1}, g_{2}, g_{3}, g_{4}, g_{5}^{1}\right\}$ and $G^{2}=\left\{g_{1}, g_{2}, g_{3}, g_{4}, g_{5}^{2}, g_{6}, g_{7}\right\}$.

Lemma 9. The sets of functions $G^{1}$ and $G^{2}$ are ECT-systems on the interval $(0, \infty)$.

Proof. To prove the statement we compute the Wronskians

$$
\begin{aligned}
& W_{1}(u)=g_{1}(u), \quad W_{2}(u)=W\left(g_{1}, g_{2}\right)(u), W_{3}(u)=W\left(g_{1}, g_{2}, g_{3}\right)(u), \\
& W_{4}(u)=W\left(g_{1}, g_{2}, g_{3}, g_{4}\right)(u), W_{5}^{1}(u)=W\left(g_{1}, g_{2}, g_{3}, g_{4}, g_{5}^{1}\right)(u), \\
& W_{5}^{2}(u)=W\left(g_{1}, g_{2}, g_{3}, g_{4}, g_{5}^{2}\right)(u), W_{6}(u)=W\left(g_{1}, g_{2}, g_{3}, g_{4}, g_{5}^{2}, g_{6}\right)(u), \text { and } \\
& W_{6}(u)=W\left(g_{1}, g_{2}, g_{3}, g_{4}, g_{5}^{2}, g_{6}, g_{7}\right)(u) .
\end{aligned}
$$

So

$$
\begin{aligned}
& W_{1}(u)=1, \\
& W_{2}(u)=2 u, \\
& W_{3}(u)=16 u^{3}, \\
& W_{4}(u)=\frac{16 u}{\left(2+u^{2}\right)^{2}}\left(P_{1}(u)+P_{2}(u) \arccos \left(\frac{u}{\sqrt{2+u^{2}}}\right)\right), \\
& W_{5}^{1}(u)=-\frac{6144 \sqrt{2} u^{3}}{\left(2+u^{2}\right)^{3}},
\end{aligned}
$$




$$
\begin{aligned}
& W_{5}^{2}(u)=-\frac{6144 \sqrt{2} \pi u^{3}}{\left(2+u^{2}\right)^{3}} \\
& W_{6}(u)=\frac{-12288 \pi u}{\left(2+u^{2}\right)^{6}}\left(Q_{1}(u)+Q_{2}(u) \arcsin \left(\frac{u}{\sqrt{2+u^{2}}}\right)\right), \quad \text { and } \\
& W_{7}(u)=-\frac{14495514624 \pi^{2} u^{3}\left(11+u^{2}\right)}{\left(2+u^{2}\right)^{8}}
\end{aligned}
$$

where

$$
\begin{aligned}
P_{1}(u)= & \sqrt{2} u\left(12+4 u^{2}+3 u^{4}\right) \\
P_{2}(u)= & 3\left(2-u^{2}\right)\left(2+u^{2}\right)^{2} \\
Q_{1}(u)= & 144 \sqrt{2} \pi-288 u-336 \sqrt{2} \pi u_{7}^{2} 20 u^{3}+1656 \sqrt{2} \pi u^{4}+14584 u^{5}+5760 \sqrt{2} \pi u^{6} \\
& +14700 u^{7}+4305 \sqrt{2} \pi u^{8}+3780 u^{9}+945 \sqrt{2} \pi u^{10}, \quad \text { and } \\
Q_{2}(u)= & 6 \sqrt{2}\left(2+u^{2}\right)^{2}\left(12-40 u^{2}+175 u^{4}+315 u^{6}\right) .
\end{aligned}
$$

Clearly $W_{1}(u) \neq 0, W_{2}(u) \neq 0, W_{3}(u) \neq 0, W_{5}^{1}(u) \neq 0, W_{5}^{2}(u) \neq 0$ and $W_{7}(u) \neq 0$ for $u>0$. To see that the function $W_{4}(u)$ does not vanish for any $u>0$ we shall prove that

$$
\tilde{P}(u)=P_{1}(u)+P_{2}(u) \arccos \left(\frac{u}{\sqrt{2+u^{2}}}\right)
$$

is an increasing function. Computing its derivative we have

$$
P^{\prime}(u)=6 u\left(\sqrt{2} u\left(2+3 u^{2}\right)+\left(3 u^{4}+4 u^{2}-4\right) \arcsin \left(\frac{u}{\sqrt{2+u^{2}}}\right)\right) .
$$

It is easy to see that $\left(3 u^{4}+4 u^{2}-4\right)$ is increasing. So $\tilde{P}^{\prime}(u)$ is also a increasing function for $u>0$, because it is sums and products of increasing functions. Since $\tilde{P}^{\prime}(0)=0$ it follows that $\tilde{P}^{\prime}(u)>0$ for every $u>0$. This implies that $\tilde{P}(u)$ is an increasing function for $u>0$. Again, since $\tilde{P}(0)=0$ it follows that $\tilde{P}(u)>0$ for every $u>0$. Thus $W_{4}(u) \neq 0$ for $u>0$.

To see that the function $W_{6}(u)$ does not vanish for any $u>0$ we shall prove that

$$
\tilde{Q}(u)=Q_{1}(u)+Q_{2}(u) \arcsin \left(\frac{u}{\sqrt{2+u^{2}}}\right)
$$

is a positive function for $u>0$. From Descartes Theorem the polynomials $Q_{1}$ and $Q_{2}$ have at most 2 zeros, and 1 minimum or maximum. Numerically we find $u_{1} \approx 0.247$ and $u_{2} \approx$ 0.269 as the minimums for $Q_{1}$ and $Q_{2}$ respectively. So $\tilde{Q}(u)$ is an increasing function for $u>\max \left\{u_{1}, u_{2}\right\}$. Finally it is easy to see that $\tilde{Q}(u)>0$ for $0<u \leq \max \left\{u_{1}, u_{2}\right\}$. Thus $W_{6}(u) \neq 0$ for $u>0$. Hence the lemma is proved.

Proof of Proposition 5. Consider system (11). Proceeding with the change of variables $x=$ $r \cos \theta$ and $y=r \sin \theta$, and taking $\theta$ as the new time, system (11) becomes

$$
r^{\prime}=\left\{\begin{array}{l}
A(\theta, r) \quad \text { if } \quad r \sin ^{2} \theta+\sin \theta-r>0, \\
B(\theta, r) \quad \text { if } \quad r \sin ^{2} \theta+\sin \theta-r<0 .
\end{array}\right.
$$


where

$$
\begin{aligned}
A(\theta, r)= & -p_{20}^{1} r^{2} \cos ^{3} \theta-r \cos ^{2} \theta\left(p_{10}^{1}+\left(p_{11}^{1}+q_{20}^{1}\right) r \sin \theta\right) \\
& -\cos \theta\left(p_{00}^{1}+r \sin \theta\left(p_{01}^{1}+q_{10}^{1}+\left(p_{02}^{1}+q_{1}^{11}\right) r \sin \theta\right)\right) \\
& -\sin \theta\left(q_{00}^{1}+r \sin \theta\left(q_{01}^{1}+q_{02}^{1} r \sin \theta\right)\right), \\
B(\theta, r)= & -r_{20}^{1} r^{2} \cos ^{3} \theta-r \cos ^{2} \theta\left(r_{10}^{1}+\left(r_{11}^{1}+s_{20}^{1}\right) r \sin \theta\right) \\
& -\cos \theta\left(r_{00}^{1}+r \sin \theta\left(r_{01}^{1}+s_{10}^{1}+\left(r_{02}^{1}+s_{1}^{11}\right) r \sin \theta\right)\right) \\
& -\sin \theta\left(s_{00}^{1}+r \sin \theta\left(s_{01}^{1}+s_{02}^{1} r \sin \theta\right)\right) .
\end{aligned}
$$

Clearly hypothesis ( $H a 1)$ holds for system (29).

Given

$$
\theta_{1}(r)=\arcsin \left(\frac{u}{\sqrt{2+u^{2}}}\right) \quad \text { and } \quad \theta_{2}(r)=\pi-\arcsin \left(\frac{u}{\sqrt{2+u^{2}}}\right),
$$

we have that for $r>0, r \sin ^{2} \theta+\sin \theta-r>0$ if and only $0 \leq \theta<\theta_{1}(r)$ and $\theta_{2}(r)<\theta \leq 2 \pi$; and $r \sin ^{2} \theta+\sin \theta-r<0$ if and only if $\theta_{1}(r)<\theta<\theta_{2}(r)$.

Let $\tilde{h}(\theta, r)=r \sin ^{2} \theta+\sin \theta-r$, thus the set of discontinuity of system (29) is given by $\tilde{\Sigma}=\tilde{h}^{-1}(0)=\left\{\left(\theta_{1}(r), r\right): r>0\right\} \cup\left\{\left(\theta_{2}(r), r\right): r>0\right\}$. Since

$$
\begin{aligned}
& \left\langle\nabla \tilde{h}\left(\theta_{1}(r), r\right),\left(1, A\left(\theta_{1}(r), r\right)\right)\right\rangle\left\langle\nabla \tilde{h}\left(\theta_{1}(r), r\right),\left(1, B\left(\theta_{1}(r), r\right)\right)\right\rangle=\frac{\left(1+4 r^{2}\right)\left(-1+\sqrt{1+4 r^{2}}\right)}{2 r^{2}}, \\
& \left\langle\nabla \tilde{h}\left(\theta_{2}(r), r\right),\left(1, A\left(\theta_{2}(r), r\right)\right)\right\rangle\left\langle\nabla \tilde{h}\left(\theta_{2}(r), r\right),\left(1, B\left(\theta_{2}(r), r\right)\right)\right\rangle=\frac{\left(1+4 r^{2}\right)\left(-1+\sqrt{1+4 r^{2}}\right)}{2 r^{2}},
\end{aligned}
$$

we conclude that $\tilde{\Sigma}$ has only crossing regions. So hypothesis (H2a) holds for system (29).

Taking $r=u \sqrt{2+u^{2}} / 2$ and computing the averaged function $f_{1}$ we obtain

$$
f_{1}(u)=k_{1} g_{1}(u)+k_{2} g_{2}(u)+k_{3} g_{3}(u)+k_{4} g_{4}(u)+k_{5} g_{5}^{1}(u)
$$

where

$$
\begin{aligned}
& k_{1}=24 \sqrt{2}\left(q_{00}^{1}-s_{00}^{1}\right), \\
& k_{2}=2 \sqrt{2}\left(-3 p_{10}^{1}+2 p_{11}^{1}+3 q_{01}^{1}+4 q_{02}^{1}+2 q_{20}^{1}+3 r_{10}^{1}-2 r_{11}^{1}-3 s_{01}^{1}-4 s_{02}^{1}-2 s_{20}^{1}\right), \\
& k_{3}=6 \sqrt{2}\left(q_{02}^{1}-s_{02}^{1}\right) \\
& k_{4}=-6\left(p_{10}^{1}+q_{01}^{1}-r_{10}^{1}-s_{01}^{1}\right), \\
& k_{5}=-3\left(p_{10}^{1}+q_{01}^{1}-r_{10}^{1}-s_{01}^{1}\right) .
\end{aligned}
$$

So from Lemma 9 and Theorem A the proof follows.

Proof of Proposition 6. In order to apply Theorem B to system (29) we have to guarantee that $f_{1}(u) \equiv 0$. By the linearity of the set of functions $G^{1}, f_{1}(u) \equiv 0$ if and only if $k_{i}=0$ for $i=$ $1,2, \ldots, 5$. Thus assuming that $k_{i}=0$ for $i=1,2, \ldots, 5$, it is easy to see, using some algebraic manipulator as Mathematica or Maple, that the statement $\left\langle\nabla h\left(\theta_{1}(r), r\right),\left(s, y_{1}\left(\theta_{1}(r), t\right)\right)\right\rangle=0$ implies $s=0$ holds if and only if the conditions $\mathcal{B}$ holds. So assuming conditions $\mathcal{B}$ the hypothesis $(H b 2)$ holds.

Taking $r=u \sqrt{2+u^{2}} / 2$ and computing the averaged function $f_{1}$ we obtain

$$
f_{2}(u)=k_{1} g_{1}(u)+k_{2} g_{2}(u)+k_{3} g_{3}(u)+k_{4} g_{4}(u)+k_{5} g_{5}^{2}(u)+k_{6} g_{6}(u)+k_{6} g_{6}(u) .
$$

Hence from Lemma 9 and Theorem B the proof follows. 


\section{Appendix: Basic Results on the Brouwer Degree}

In this appendix we present the existence and uniqueness result from the degree theory in finite dimensional spaces. We follow the Browder's paper [8], where are formalized the properties of the classical Brouwer degree. We also present some results that we shall need for proving the main results of this paper.

Theorem 10. Let $X=\mathbb{R}^{n}=Y$ for a given positive integer $n$. For bounded open subsets $V$ of $X$, consider continuous mappings $f: \bar{V} \rightarrow Y$, and points $y_{0}$ in $Y$ such that $y_{0}$ does not lie in $f(\partial V)$ (as usual $\partial V$ denotes the boundary of $V$ ). Then to each such triple $\left(f, V, y_{0}\right)$, there corresponds an integer $d\left(f, V, y_{0}\right)$ having the following three properties.

(i) If $d\left(f, V, y_{0}\right) \neq 0$, then $y_{0} \in f(V)$. If $f_{0}$ is the identity map of $X$ onto $Y$, then for every bounded open set $V$ and $y_{0} \in V$, we have

$$
d\left(\left.f_{0}\right|_{V}, V, y_{0}\right)= \pm 1
$$

(ii) (Additivity) If $f: \bar{V} \rightarrow Y$ is a continuous map with $V$ a bounded open set in $X$, and $V_{1}$ and $V_{2}$ are a pair of disjoint open subsets of $V$ such that

$$
y_{0} \notin f\left(\bar{V} \backslash\left(V_{1} \cup V_{2}\right)\right),
$$

then,

$$
d\left(f_{0}, V, y_{0}\right)=d\left(f_{0}, V_{1}, y_{0}\right)+d\left(f_{0}, V_{1}, y_{0}\right) .
$$

(iii) (Invariance under homotopy) Let $V$ be a bounded open set in $X$, and consider a continuous homotopy $\left\{f_{t}: 0 \leq t \leq 1\right\}$ of maps of $\bar{V}$ in to $Y$. Let $\left\{y_{t}: 0 \leq t \leq 1\right\}$ be a continuous curve in $Y$ such that $y_{t} \notin f_{t}(\partial V)$ for any $t \in[0,1]$. Then $d\left(f_{t}, V, y_{t}\right)$ is constant in $t$ on $[0,1]$.

Theorem 11. The degree function $d\left(f, V, y_{0}\right)$ is uniquely determined by the conditions of Theorem 10 .

For the proofs of Theorems 10 and 11 see [8].

Proposition 12. We consider the continuous functions $f_{i}: \bar{V} \rightarrow \mathbb{R}^{n}$, for $i=0,1, \ldots, k$, and $f, g, r: \bar{V} \times\left[\varepsilon_{0}, \varepsilon_{0}\right] \rightarrow \mathbb{R}^{n}$, given by

$$
\begin{gathered}
g(\cdot, \varepsilon)=f_{1}(\cdot)+\varepsilon f_{2}(\cdot)+\varepsilon^{2} f_{3}(\cdot)+\cdots+\varepsilon^{k-1} f_{k}(\cdot), \\
f(\cdot, \varepsilon)=g(\cdot, \varepsilon)+\varepsilon^{k} r(\cdot, \varepsilon) .
\end{gathered}
$$

Assume that $g(z, \varepsilon) \neq 0$ for all $z \in \partial V$ and $\varepsilon \in\left[-\varepsilon_{0}, \varepsilon_{0}\right]$. If for $|\varepsilon|>0$ sufficiently small $d_{B}\left(f(\cdot, \varepsilon), V, y_{0}\right)$ is well defined, then

$$
d_{B}\left(f(\cdot, \varepsilon), V, y_{0}\right)=d_{B}\left(g(\cdot, \varepsilon), V, y_{0}\right) .
$$

For a proof of Proposition 12 see Lemma 2.1 in [9].

\section{ACKNowledgements}

The first author is partially supported by a MINECO/FEDER grant MTM2008-03437, an AGAUR grant number 2009SGR-0410, an ICREA Academia, FP7-PEOPLE-2012-IRSES316338 and 318999, and FEDER-UNAB10-4E-378. The second author is partially supported by a FAPESP-BRAZIL grant $2012 / 20884-8$. The third author is partially supported by a FAPESP-BRAZIL grant 2012/10231-7. The authors are also supported by the joint project CAPES-MECD grant PHB-2009-0025-PC. 


\section{REFERENCES}

[1] A.A. Andronov, A.A. Vitt and S.E. Khaikin, Theory of oscillators, International Series of Monographs In Physics 4, Pergamon Press, 1966.

[2] E. A. Barbashin, Introduction to the Theory of Stability (T. Lukes, Ed.), Noordhoff, Groningen, 1970.

[3] A.D. Bazykin, Nonlinear Dynamics of Interacting Populations, River-Edge, NJ, World Scientific, 1998.

[4] I.S. Berezin And N.P. Zhidkov, Computing Methods, Volume II, Pergamon Press, Oxford, 1964.

[5] N.N. Bogoliubov, On some statistical methods in mathematical physics, Izv. vo Akad. Nauk Ukr. SSR, Kiev, 1945.

[6] N. N. Bogoliubov And N. KRylov, The application of methods of nonlinear mechanics in the theory of stationary oscillations, Publ.8 of the Ukrainian Acad. Sci. Kiev, 1934.

[7] B. Brogliato, Nonsmooth Mechanics, New York, Springer-Verlag, 1999.

[8] F. Browder, Fixed point theory and nonlinear problems, Bull. Amer. Math. Soc. 9 (1983), 1-39.

[9] A. Buica And J. Llibre, Averaging methods for finding periodic orbits via Brouwer degree, Bulletin des Sciences Mathemàtiques 128 (2004), 7-22.

[10] P. Fatou, Sur le mouvement d'un systàme soumis à des forces à courte période, Bull. Soc. Math. France 56 (1928), 98-139.

[11] A. F. Filippov, Differential Equations with Discontinuous Righthand Side, Mathematics and Its Applications, Kluwer Academic Publishers, Dordrecht, 1988.

[12] C. Henry, Differential equations with discontinuous righthand side for planning procedure, J. Econom. Theory 4 (1972), 541-551.

[13] Т. Iто, A Filippov solution of a system of differential equations with discontinuous right-hand sides, Economic Letters 4 (1979), 349-354.

[14] S.J. Karlin And W.J. Studden, T-Systems: With Applications in Analysis and Statistics, Pure Appl. Math., Interscience Publishers, New York, London, Sidney, 1966.

[15] V. Krivan, On the Gause predator-prey model with a refuge: A fresh look at the history, J. Theoretical Biology 274 (2011), 67-73.

[16] J. Llibre, D.D. Novaes And M.A. Teixeira, Averaging methods for studying the periodic orbits of discontinuous differential systems, arXiv:1205.4211 [math.DS], http://arxiv.org/abs/1205.4211.

[17] J. Llibre And G. Swirszcz, On the limit cycles of polynomial vector fields, Dyn. Contin. Discrete Impuls. Syst. 18 (2011), 203-214.

[18] N. Minorski, Nonlinear Oscillations, Van Nostrand, New York, 1962.

[19] J. A. Sanders, F. Verhulst and J. Murdock, Averaging method in nonlinear dynamical systems, Appl. Math. Sci., vol. 59, Springer, New York, 2007.

[20] M.A. TeIXeIRA, Perturbation theory for non-smooth systems, Encyclopedia of Complexity and Systems Science 22, Springer, New York, 2009, pp 6697-6719.

[21] F. Verhulst, Nonlinear Differential Equations and Dynamical Systems, Universitext, Springer, 1991.

[22] VArious, Special issue on dynamics and bifurcations of nonsmooth systems, Physica D 241 (2012), 18252082 .

${ }^{1}$ Departament de Matematiques, Universitat Autònoma de Barcelona, 08193 Bellaterra, Barcelona, Catalonia, Spain

E-mail address: jllibre@mat.uab.cat

2 Departamento de Física, Química e Matematica, Universidade Federal de São Carlos, 18052-780, SOROCABA, SP, BrazIL

E-mail address: anamereu@ufscar.br

3 Departamento de Matematica, Universidade Estadual de Campinas, Caixa Postal 6065, 13083859, Campinas, SP, Brazil

E-mail address: ddnovaes@gmail.com 